\title{
The effect of accounting for biarticularity in hip flexor and hip extensor joint torque representations
}

\author{
M.G.C. Lewis ${ }^{1}$, M.R. Yeadon², M.A. King ${ }^{2}$ \\ 1School of Science and Technology, Nottingham Trent University, Nottingham NG11 8NS, UK \\ ${ }^{2}$ School of Sport, Exercise and Health Sciences, Loughborough University, Leicestershire, LE11 3TU, UK
}

\begin{abstract}
Subject-specific torque-driven models have ignored biarticular effects at the hip. The aim of this study was to establish the contribution of monoarticular hip flexors and hip extensors to total hip flexor and total hip extensor joint torques for an individual and to investigate whether torque-driven simulation models should consider incorporating biarticular effects at the hip joint. Maximum voluntary isometric and isovelocity hip flexion and hip extension joint torques were measured for a single participant together with surface electromyography. Single-joint and two-joint representations were fitted to the collected torque data and used to determine the maximum voluntary joint torque capacity. When comparing two-joint and single-joint representations, the single-joint representation had the capacity to produce larger maximum voluntary hip flexion torque (larger by around $9 \%$ of maximum torque) and smaller maximum voluntary hip extension torque (smaller by around $33 \%$ of maximum torque) with the knee extended. Considering the range of kinematics found for jumping movements, the single-joint hip flexors had the capacity to produce around $10 \%$ additional torque, while the single joint hip extensors had about $70 \%$ of the capacity of the two-joint representation. Two-joint representations may overcome an over-simplification of single-joint representations by accounting for biarticular effects, while building on the strength of determining subject-specific parameters from measurements on the participant.
\end{abstract}

Keywords: computer simulation, joint torque, biarticular muscle

\section{HIGHLIGHTS}

- Contribution of monoarticular hip flexors and extensors to total hip torque.

- Measured maximum voluntary hip flexion and hip extension joint torques.

- Single-joint and two-joint representations fitted.

- Two-joint representations may overcome a limitation of single-joint representations.

- Two-joint representations are expected to improve the biofidelity of torque-driven models.

\section{INTRODUCTION}

Whole body forward dynamics simulation models are typically either torquedriven or muscle-driven. Muscle-driven models enable the study of relatively complex systems (Neptune and Hull, 1998; Anderson and Pandy, 1999) and are necessary when the role of individual muscles are investigated (Pandy et al., 1990; van Soest et al., 1993; Jacobs et al., 1996; Bohm et al., 2006). Muscle-driven models typically require that many of the individual muscle strength parameters are selected from multiple literature sources and therefore may not in these cases be representative of either an individual or a generic human. In some cases, authors have looked to avoid introducing multiple sources of error into complex individual muscle driven model by attempting to establish a cohesive data set from which parameters may be derived from a single-source. In the case of the study by Arnold et al. (2010) a data set was utilised which enabled multiple individual parameters to be collected from a single source where multiple muscle-tendon parameters were identified for the same cadaver. Their simulation model was intended to study the 
role and function of human muscle and tendon, which it was well-placed to achieve. However the same method is not appropriate for identifying a global optimum solution for movement since there is no kinematic and kinetic data against which to evaluate the model.

Some authors using an individual muscle modelling approach have sought to minimise simulation model errors by reducing the complexity of the design by grouping muscles together which have similar operational roles, for example by considering the three hamstrings as a single hamstring muscle (van Soest et al., 1993). This reduced parameter set might then be be scaled to represent an individual or small subset of participants (Domire and Challis, 2007), enabling a simulation model to investigate muscle and tendon roles whilst also retaining the possibility to evaluate the movement solution against participant data. If the predominant need for a simulation model is to investigate aspects of technique, with less dependence on understanding this technique at the level of individual muscle contributions, then a lumped muscle-modelling approach will provide the necessary solution and further reduce the complexity of the model and potential sources of error. Torque-driven models have the advantage that it is possible to determine subject-specific strength parameters from measurements made using an isovelocity dynamometer and to then evaluate the whole-body simulation model against a recorded performance (King et al., 2006; Allen et al., 2013). In order that the model adequately produces a realistic performance, it is the generation of realistic net joint torques that is required, rather than any in depth understanding of the individual role of muscles. As a consequence fewer muscle and tendon parameters are required.

Historically torque-driven models have produced good representations for a number of activities (e.g. tumbling takeoff, King and Yeadon, 2004; high jumping takeoff, Wilson et al., 2007; triple jumping takeoffs, Allen et al., 2010), with the torque at a joint calculated only from single joint kinematics (monarticular representations). In these models the effect of biarticularity and changes in length at a secondary joint are ignored. Thus it is not clear what advantage incorporating biarticular representations may have and in which circumstances they may be beneficial. More recently, subject-specific combined monoarticular and biarticular torque generator parameters at the ankle (Lewis et al., 2012) and knee joint (King et al., 2012) have been derived from isovelocity torque measurements to address the issue of biarticular effects. For ankle plantar flexor joint torque it was demonstrated that if the knee was flexed by more than $40^{\circ}$ a two-joint representation was appropriate (Lewis et al., 2012). Similarly, at the knee the total flexor and total extensor joint torque representations were improved when monoarticular and biarticular components were used as opposed to just using the knee joint kinematics alone (King et al., 2012). The additional complexity in this two-joint lumped approach, enables groups of muscles operating with similar function, to be simplified into two groups, those which are affected by one joint and those which are affected by two joints, but still without the detail being at the individual muscle level.

At the hip, previous subject-specific torque-driven forward-dynamics simulation models have calculated maximum voluntary hip flexor and extensor torques using the kinematics of the hip alone (e.g. tumbling; King and Yeadon, 2004, diving; King et al., 2005, jumping for height; King et al., 2006). The monoarticular muscle contributions to total hip flexor torque and total hip extensor torque may be in the region of $62 \%$ and $46 \%$ respectively based upon physiological cross-sectional area, pennation 
angle and moment arms in the literature assuming equal activation of all muscles (Appendix A). Biarticular hip-knee muscles make a substantial contribution to torque at the hip joint, although it is not clear if including the biarticular contribution within a monoarticular representation at the hip is appropriate for all movements. The maximum knee flexor and knee extensor torques exerted by the two-joint knee-hip torque generators of King et al. (2011) can be used to calculate the torques which would be exerted by the same biarticular components at their secondary joint (in this case the hip) by using the ratio of moment arms previously established through optimisation (Appendix B). Figure 1 shows the hip flexor and hip extensor torques generated by the biarticular knee extensor and biarticular knee flexor torque generators respectively. Here the activation is assumed to be maximal and the knee angle fixed at one of three joint angles throughout the functional joint range. Biarticular hip flexion and hip extension representations would contribute the largest hip torques when the biarticular muscles were at their longest lengths and contracting eccentrically (hip flexion: knee flexed, hip extended Figure 1a, b, c; hip extension: knee extended, hip flexed, Figure 1d, e, f). The biarticular hip torque would vary substantially as a function of knee angle; for biarticular hip flexion the eccentric torque would range from $14 \mathrm{Nm}$ when the knee was flexed through to $0 \mathrm{Nm}$ if the knee was within $42^{\circ}$ of full extension (Figure 1a, b, c). For biarticular hip extension the maximum eccentric torque would equate to $95 \mathrm{Nm}$, with biarticular torques unable to be exerted for posterior knee joint angles of $44^{\circ}$ to full flexion (Figure $1 \mathrm{~d}, \mathrm{e}, \mathrm{f}$ ). It is clear that were a biarticular knee-hip torque generator to be making a contribution to the net hip flexor or hip extensor torque, then the capacity of the hip joint to exert torque would differ from a single monoarticular representation of hip torques measured for a fixed knee joint angle if there were multi-joint kinematics.

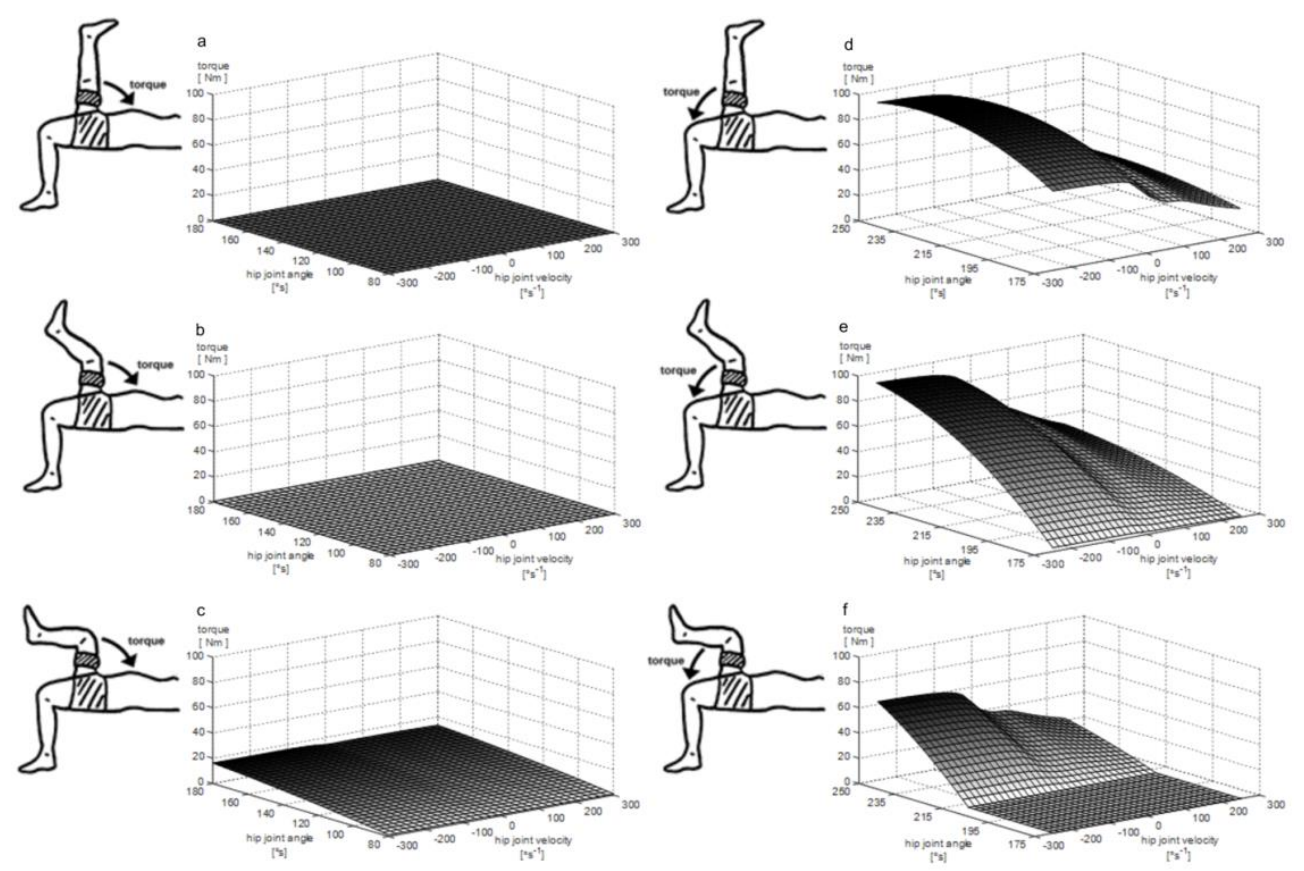

Figure 1. Biarticular torque contribution to hip torques for three different knee joint angles. Hip flexor biarticular torque contribution for knee joint angles of a) $180^{\circ}$ (full extension), b) $135^{\circ}$ (midrange), c) $90^{\circ}$ (flexed). Hip extensor biarticular torque contribution for knee joint angles of d) $180^{\circ}$ (full extension), e) $135^{\circ}$ (mid-range), f) $90^{\circ}$ (flexed). Parameters derived from King et al. 2012. Hip flexion joint angle corresponds to the anterior hip joint angle, and hip extension joint angle to the posterior hip joint angle; angles measured between the line of the trunk and thigh. 
Therefore the aim of this study was to establish the contribution of monoarticular hip flexors and hip extensors to total hip flexor and total hip extensor joint torques for an individual using previously derived parameters for biarticular knee-hip torque generators and to determine the magnitude of the difference between a single-joint and two-joint representation of hip torques.

\section{METHODS}

\subsection{Participants}

Maximum voluntary isometric and isovelocity hip flexion and hip extension joint torques were measured for a single participant (28 year old male volleyball player, height $1.74 \mathrm{~m}$, mass $79.2 \mathrm{~kg}$ ) with experience of strength measurement on isovelocity dynamometers. The participant gave informed consent for the procedures in accordance with a protocol approved by the Loughborough University ethics committee.

\subsection{Procedures}

Dynamometer torque, crank angle and crank velocity data were measured using a Contrex multi-joint isovelocity dynamometer (CMV AG, Switzerland) sampling at a frequency of $256 \mathrm{~Hz}$. All trials were performed with a foam wedge strapped firmly behind the knee joint to maintain a constant knee joint angle of approximately $90^{\circ}$. The standard deviation of the knee joint angle for Hip flexion and Hip extension trials was $3.3^{\circ}$ and $2.2^{\circ}$ respectively. The participant was lying in a supine position and was strapped firmly to the dynamometer with the joint and crank centres aligned under load to reduce freedom in the system and to minimise movement of the joint axis relative to the crank axis (Figure 2).

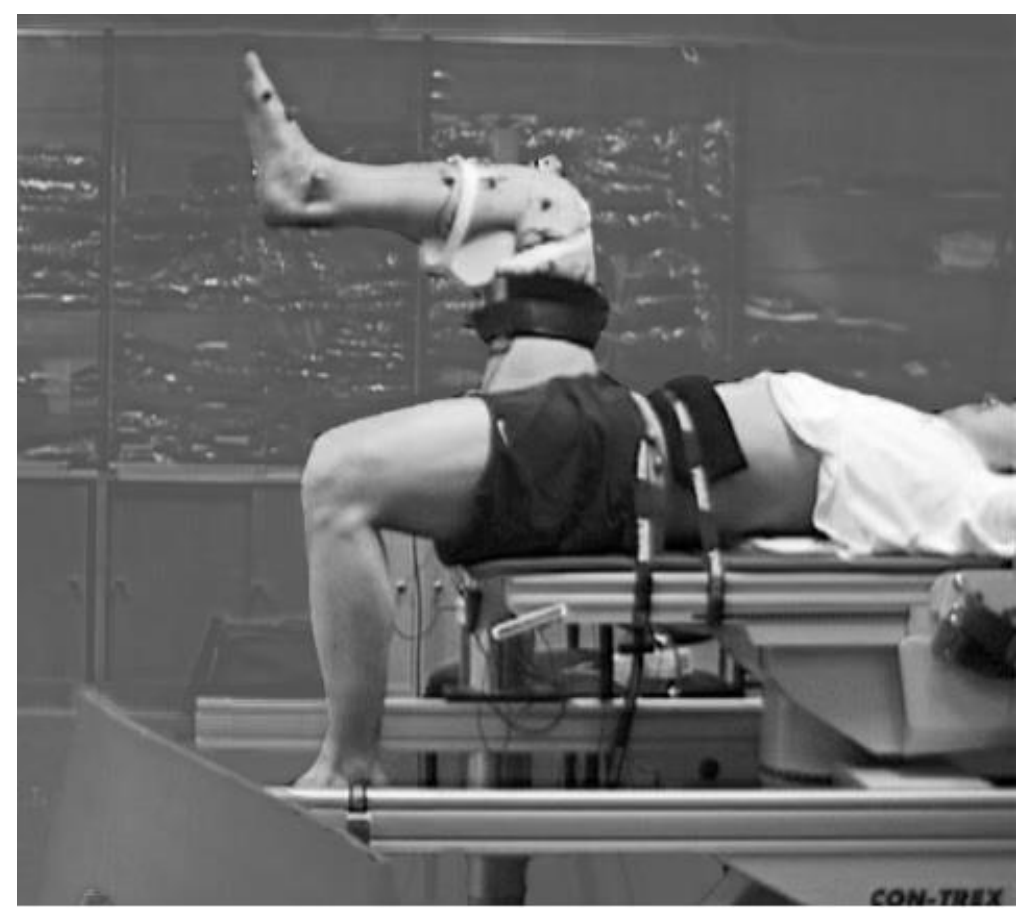

Figure 2. Participant positioning on the dynamometer.

Maximum voluntary isometric torque was measured for seven hip flexion and seven hip extension trials, each lasting five seconds. The hip joint angles used for 
isometric trials were distributed throughout the functional flexion-extension range of motion. Maximum voluntary isovelocity torque was measured for a series of 3 repeated concentric-eccentric trials over a range of velocities from $50^{\circ} \mathrm{s}^{-1}$ to $300^{\circ} \mathrm{s}^{-1}$ in $50^{\circ} \mathrm{s}^{-1}$ intervals. Trials were not randomised to ensure the participant was adequately prepared to produce maximum torque for each trial. The first repetition in the series of three was disregarded to ensure the contractions which were analysed were from a pre-tensioned state. The concentric and eccentric contractions selected for analysis were those with the largest overall torque. The knee and hip joint centres were calculated throughout each trial from $14 \mathrm{~mm}$ spherical reflective markers that were placed around each joint and tracked using a 16 camera (M2 MCam) Vicon Motion Analysis System (OMG Plc, Oxford, UK) operating at $300 \mathrm{~Hz}$ (King et al., 2012). Hip flexion-extension angles were calculated throughout each trial from the joint centre locations using Cardan rotations (King et al., 2012).

Dynamometer torque, crank angle and angular velocity and hip joint angle data were filtered using a zero lag $12 \mathrm{~Hz}$ low pass, fourth order Butterworth filter, where the cut-off frequency was established from a residual analysis (Winter, 1990). Dynamometer torque and crank data were resampled at $300 \mathrm{~Hz}$ using a quintic interpolating spline and were synchronised with the hip joint angle data using linear correlation with the crank angle data (King et al., 2012). Torque measurements were corrected for the weights of the crank and the participant's limb, and any additional passive torques present throughout the crank angle range (Pavol and Grabiner, 2000). Isovelocity torque measurements were selected from periods when the crank velocity was between $95 \%$ and $105 \%$ of the preset crank velocity, removing periods of overshoot (Schwartz et al., 2010) and correcting for inertial torques (King et al., 2012).

Biarticular knee flexion and knee extension strength parameters for the participant were calculated in a previous study (Table 1; King et al., 2012), including the ratio $\mathrm{R}$ of moment arms at the hip and knee joints for the biarticular torque generators. The maximum hip flexion and hip extension torques due to the biarticular knee flexion and knee extension muscles were calculated using these parameters by multiplying the maximal biarticular knee flexion and knee extension torque (function of hip and knee angle) by the equivalent moment arm ratio (see Appendix B and Fig. 3 for further details). Surface electromyography (EMG) was used to establish the activation state of the biarticular rectus femoris, biceps femoris and semitendinosus muscles during maximal voluntary isometric hip flexion, hip extension, knee flexion and knee extension using an active bipolar surface electrode system (Biovision: Wehrheim, Germany), sampled at $2400 \mathrm{~Hz}$. Surface EMG measurements were band pass filtered $(10-500 \mathrm{~Hz})$ and notch filtered at $50 \mathrm{~Hz}$ using a $4^{\text {th }}$ order zero-lag Butterworth filter. Root mean square EMG amplitude (RMSEMG) was determined for the duration of the muscle activation (Katsavelis and Threlkeld, 2014) and was normalised to the maximum agonist surface EMG measured for isometric knee flexion or knee extension trials. It was assumed that that the relationship between the force generated by a muscle and the surface EMG associated with the force has a curvilinear relationship (Kooistra et al. 2007), where for EMG measurements up to approximately $60 \%$ the force and EMG relationship is linear and after that an increase in muscle force, is not necessarily associated with a similar relative increase in muscle EMG. In this study all surface EMG measurements exceeded $60 \%$ of maximal activation $(67 \%-96 \%)$ and were within 
the non-linear region (Table 2). As a consequence and to avoid over-estimating the contribution of monoarticular torque generators it was assumed that the surface EMG for both hip flexion and hip extension indicated a $100 \%$ contribution from the biarticular components during hip flexion and hip extension. The contribution from monoarticular muscles was calculated by subtracting the active biarticular torque contribution from the measured total joint torque for both hip flexion and hip extension (Appendix B).

\begin{tabular}{ccc}
$\begin{array}{c}\text { Table 1. Two-joint biarticular torque } \\
\text { generator parameters for the knee. }\end{array}$ \\
\hline parameter & $\begin{array}{c}\text { knee } \\
\text { flexion }\end{array}$ & $\begin{array}{c}\text { knee } \\
\text { extension }\end{array}$ \\
\hline $\mathrm{T}_{\max }$ & 99.78 & 78.93 \\
$\mathrm{~T}_{0}$ & 71.27 & 56.38 \\
$\omega_{\max }$ & 18.50 & 23.39 \\
$\omega_{\mathrm{c}}$ & 6.15 & 10.3 \\
$\mathrm{k}_{2}$ & 0.26 & 0.4 \\
$\theta_{\mathrm{opt}}$ & 7.92 & 6.86 \\
$\mathrm{a}_{\mathrm{min}}$ & 0.82 & 0.64 \\
$\mathrm{~m}$ & 0.28 & 0.83 \\
$\omega_{1}$ & 1.57 & 0.61 \\
$\mathrm{R}$ & 1.19 & 0.44 \\
\hline
\end{tabular}

Nomenclature: maximum eccentric torque $\mathrm{T}_{\max }(\mathrm{Nm})$, maximum isometric torque $\mathrm{T}_{0}(\mathrm{Nm})$, maximum concentric velocity $\omega_{\max }\left(\mathrm{rad} . \mathrm{s}^{-1}\right)$, vertical asymptote $\omega_{\mathrm{c}}\left(\mathrm{rad} . \mathrm{s}^{-1}\right)$, width of torque-angle relationship k2, optimum angle, $\theta_{\mathrm{opt}}$ $(\mathrm{rad})$, minimum muscle activation, $\mathrm{a}_{\min }$, activation rate, $\mathrm{m}$ and point of inflexion $\omega_{1}\left(\mathrm{rad} . \mathrm{s}^{-1}\right)$, and moment arm ratio $\mathrm{R}$. All were derived from isokinetic dynamometer measurements made on the same participant as in this study (see King et al. (2012) and Lewis et al. (2012)).

a)

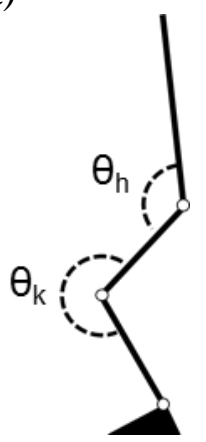

b)

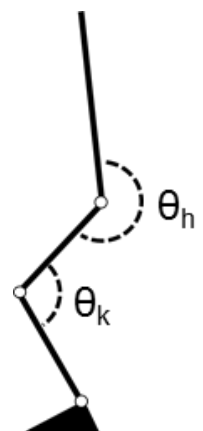

Figure 3. Angle conventions used for torque representations; a) Hip flexion and knee extension, b) Hip extension and knee flexion.

Table 2. Mean SEMG for all isometric hip extension or isometric hip flexion trials

\begin{tabular}{ccc}
\hline muscle & $\begin{array}{c}\text { hip extension } \\
{[\%]}\end{array}$ & $\begin{array}{c}\text { hip flexion } \\
{[\%]}\end{array}$ \\
\hline rectus femoris & $8.1 \pm 1.5$ & $67.0 \pm 11.7^{*}$ \\
combined hamstrings & $96.1 \pm 19.5^{*}$ & $46.1 \pm 14.7$ \\
\hline
\end{tabular}

* agonist 


\subsection{Representation of Joint Torque}

The monoarticular joint torque contribution for hip flexion and hip extension was fitted using a nine-parameter function of hip joint angle and hip joint angular velocity by minimising a weighted root mean square difference (RMSD, King et al., 2012) between the monoarticular joint torque and the nine-parameter function using the Simulated Annealing Algorithm (Corana et al., 1987). Single-joint nine-parameter representations were also determined for the total hip flexion and the total hip extension joint torque as a function of hip angle and hip angular velocity by minimising a weighted RMSD between total hip torques and a nine-parameter representation. The maximal voluntary monoarticular representation and combined monoarticular + biarticular torque representation (two-joint) were compared with the typically used single-joint torque representations by comparison of the RMSD's and by comparing the general shape of the torque-angle-angular velocity curves for both hip flexion and hip extension under the assumption of zero velocity at the knee joint. Isokinetic dynamometers are designed to enable dynamic movement for a small subset of joint kinematics, but not designed for multi-joint motion. To provide some perspective of the difference between single-joint and two joint calculations for functional dynamic tasks, the knee and hip kinematics for the participant for three two-footed jumps: squat, countermovement and broad jump were recorded (Appendix $\mathrm{C}$ ). The kinematics were used to calculate maximal voluntary joint torque capacity at the hip for both the two-joint torque representation and the traditionally used single-joint torque representation. All jump data were collected within the same 2-week period during which all joint torque measurements of the knee and hip were collected.

\section{RESULTS}

The calculated biarticular hip flexor torque calculated from the parameter values of King et al. (2012) represented up to $14 \mathrm{Nm}$ or $8 \%$ of the maximum measured total hip flexor torque of $174 \mathrm{Nm}$, whilst the biarticular hip extensor representation could contribute up to $56 \mathrm{Nm}$ or $35 \%$ of the maximum measured total hip extensor torque of $158 \mathrm{Nm}$ (Figure 1).

\begin{tabular}{|c|c|c|c|}
\hline action & $\begin{array}{l}\text { differer } \\
\text { calcula }\end{array}$ & en singl & two-joint \\
\hline & & xion & \\
\hline knee joint angle* & $180^{\circ}$ & $225^{\circ}$ & $\$ 270^{\circ}$ \\
\hline & $8.7 \%$ & $8.7 \%$ & $6.2 \%$ \\
\hline & & ension & \\
\hline knee joint angle* & $180^{\circ}$ & $135^{\circ}$ & $\$ 90^{\circ}$ \\
\hline & $33.3 \%$ & $20.3 \%$ & $4.7 \%$ \\
\hline
\end{tabular}

The single-joint nine-parameter torque representation (Figure 4a) of the total hip flexor joint torques gave a weighted RMSD of $13.9 \mathrm{Nm}$ ( $8 \%$ of the maximum hip flexion torque) and the monoarticular hip flexion torque representation (Figure 4b) 
had a weighted RMSD of $9.9 \mathrm{Nm}(6 \%)$. Some notable differences were found between the single joint and monoarticular representations in spite of the relatively small $15 \mathrm{Nm}$ difference in the peak torque generated by the two representations. In particular the optimum angle for the monoarticular torque representation occurred at a hip joint angle $27^{\circ}$ larger and the general shape of the torque-angle curve was much flatter than the single joint representation (Figure 4d; Table 4). These differences meant that each representation estimated larger torques than the other for some part of the joint range, with the single joint torque representation unable to generate torque at smaller hip joint angles. Overall at the $90^{\circ}$ knee angle used for the torque measurements, the two-joint representation (monoarticular plus biarticular torques) and the single-joint representation of hip flexion were similar with relatively small differences (RMSD $11.6 \mathrm{Nm}(6.2 \%$ of max torque)) in the net maximum voluntary torque that could be produced over a range of hip angles and angular velocities (Figure 4e). Calculating the joint torques for a knee with a mid-range angle or in a fully extended position did not alter the single-joint torque representation but did reduce the biarticular torque component in the two-joint representation (Figure 1a, b). Compared to the two-joint representation, the single-joint representation calculated larger maximum voluntary torque at the hip and the RMSD between them increased to $16.2 \mathrm{Nm}(8.7 \%)$ when the knee was extended (Table 3). For the kinematics of the three jumping movements (using the knee and hip angle time histories and assuming maximum activation), the single-joint representation had the capacity to produce an average of $10 \%$ larger hip flexion torque than the two-joint representation.

Table 4. Hip flexion torque generator parameters

\begin{tabular}{cccc}
\hline parameter & mono & $\begin{array}{c}\text { single } \\
\text { joint }\end{array}$ & $\begin{array}{c}\text { bounds } \\
(\text { LB - UB })\end{array}$ \\
\hline $\mathrm{T}_{\max }$ & 198.67 & 202.44 & $1.4\left(\mathrm{~T}_{0}\right)$ \\
$\mathrm{T}_{0}$ & 141.92 & 144.60 & $0.8-1.2\left(\mathrm{~T}_{0}\right)$ \\
$\omega_{\max }$ & 9.33 & 9.99 & $7.5-10$ \\
$\omega_{\mathrm{c}}$ & 3.89 & 3.81 & $0.15-0.5\left(\omega_{\max }\right)$ \\
$\mathrm{k}_{2}$ & 0.23 & 0.50 & $0.2-2.0$ \\
$\theta_{\mathrm{opt}}$ & 3.47 & 3.00 & $1.0-4.0$ \\
$\mathrm{a}_{\min }$ & 0.87 & 0.93 & $0.2-1.0$ \\
$\mathrm{~m}$ & 0.13 & 0.07 & $0.0-1.0$ \\
$\omega_{1}$ & 1.57 & 1.33 & $-0.5-3.0$
\end{tabular}

Nomenclature and bounds based upon: maximum eccentric torque $\mathrm{T}_{\max }(\mathrm{Nm})$ (Dudley et al.,1990; Webber and Kriellers, 1997), maximum isometric torque $\mathrm{T}_{0}(\mathrm{Nm})$, maximum concentric velocity $\omega_{\max }\left(\mathrm{rad} . \mathrm{s}^{-1}\right)$ (King et al. (2006)), vertical asymptote $\left(\omega=-\omega_{c}\right) \omega_{c}$ $\left(\right.$ rad. $\left.{ }^{-1}\right)$ (UB - Scovil and Ronsky (2006); LB - Umberger et al. (2006)), width of torque-angle relationship k2, optimum angle, $\theta_{\mathrm{opt}}$ (rad) (UB permitted outside joint range where curve may be ascending only), minimum muscle activation, amin, activation rate, $\mathrm{m}$ and point of inflexion $\omega_{1}$ (rad. $\left.\mathrm{s}^{-1}\right)$ (Amiridis et al. (1996)). 

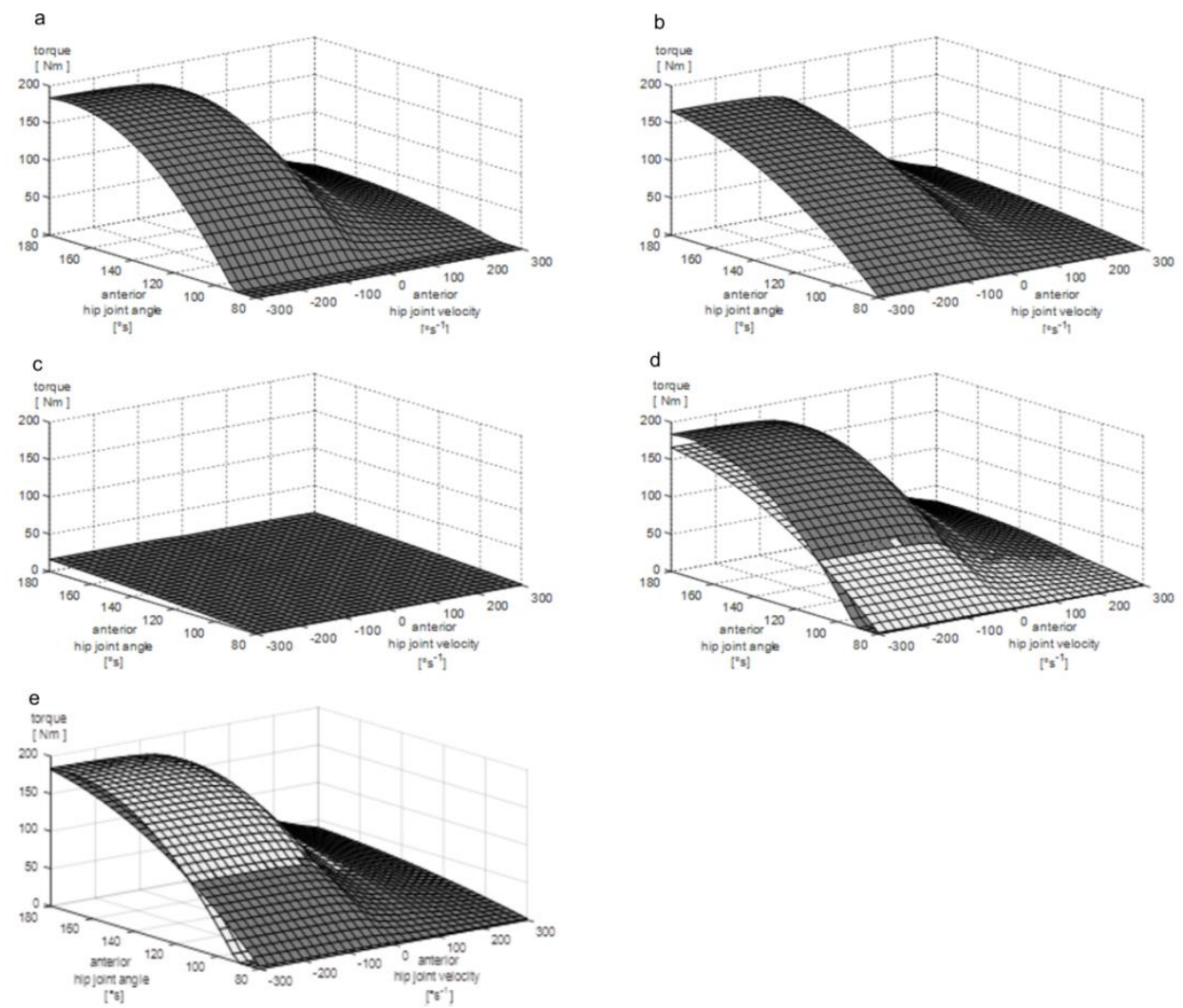

Figure 4. Hip flexion (knee angle 90) for; a) single-joint representation, b) monoarticular representation, c) biarticular representation, d) single-joint (dark shading), monoarticular representation (light shading), e) combined two-joint representation (dark shading) and single-joint representation (light shading). The hip joint angle corresponds to the anterior hip joint angle, an angle measured in an anterior direction between the line of the trunk and thigh.

The corresponding joint torque representations for the single-joint (Figure 5a) and monoarticular hip extension torques (Figure 5b) had weighted RMSDs of 10.9 $\mathrm{Nm}(7 \%$ of the maximum hip extension torque) and $11.7 \mathrm{Nm}(7 \%)$ respectively. Although the biarticular joint torque component for hip extension (maximum $55 \mathrm{Nm}$ ) was larger than for hip flexion, the monoarticular and single joint hip extensor torque representations exhibited some features similar to those for hip flexion (Figure 5; Table 5). The width parameter differed between the two representations in a way that enabled the monoarticular representation to exert larger torques than the single joint representation at smaller hip joint angles and the optimum joint angle was $17^{\circ}$ smaller for the monoarticular representation (Figure 5d; Table 5). Overall the twojoint representation and the single-joint representation were able to produce similar maximum voluntary net joint torques for the $90^{\circ}$ knee angle used for the torque measurements (Figure $5 \mathrm{e}$; RMSD $7 \mathrm{Nm} ; 4.7 \%$ of maximum torque)). Calculating the joint torques for a knee at a mid-range angle or extended position as for the hip flexors did not alter the single-joint torque representation but increased the biarticular torque component in the two-joint representation for hip extension (Figure 1d, e). Compared to the two-joint representation, the single-joint representation for hip extension calculated smaller maximum voluntary torque at the hip and the RMSD increased to $55.7 \mathrm{Nm}$ (33.3\% of maximum torque) when the knee was extended. For the kinematics of the three jumping movements (using the knee and hip angle time histories and assuming maximum activation), the two-joint representation had the capacity to produce on average $30 \%$ larger hip extension torques than the singlejoint representation. 

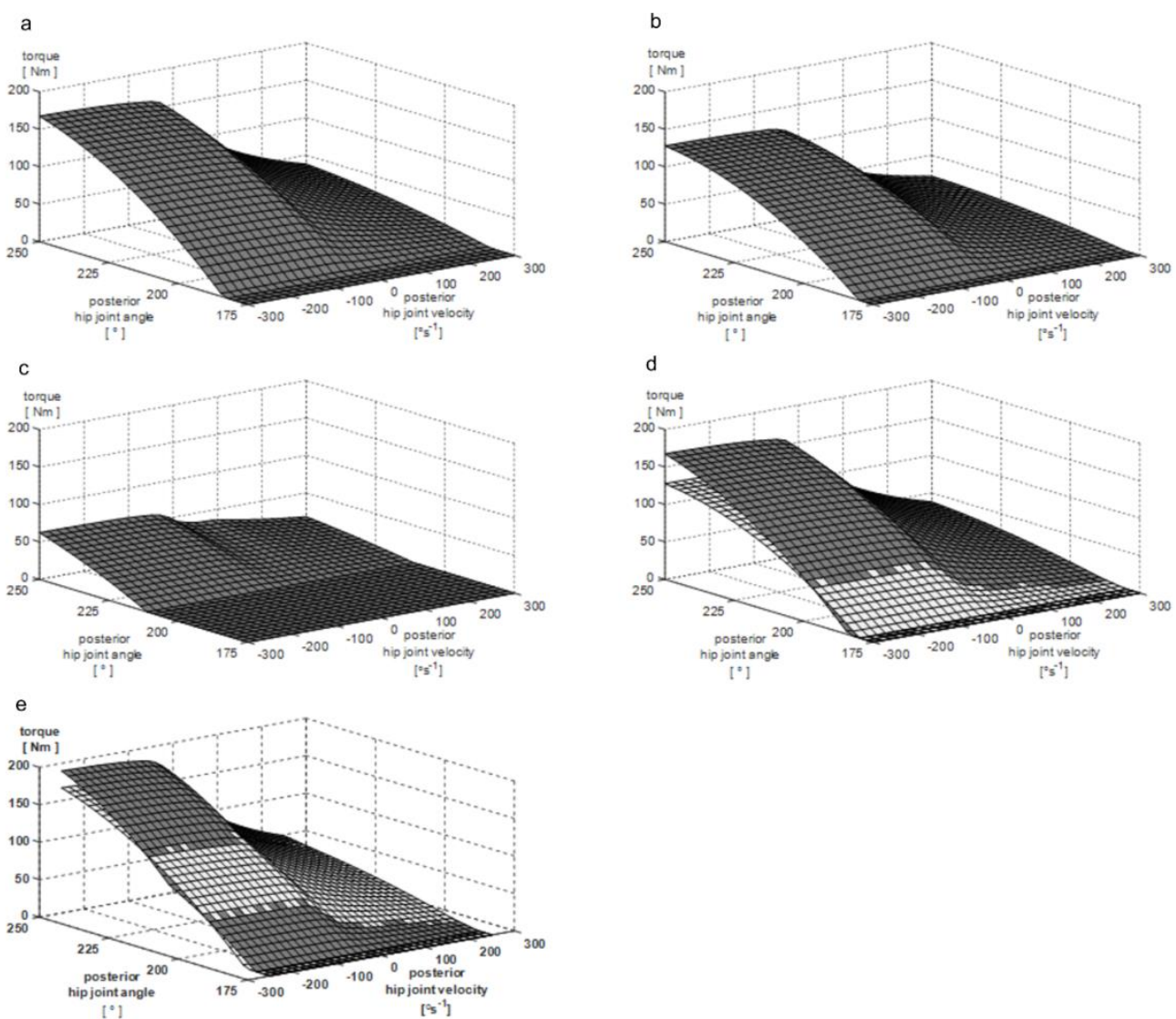

Figure 5. Hip extension (knee angle 90); a) single-joint representation, b) monoarticular representation, c) biarticular representation, d) single-joint (dark shading), monoarticular representation (light shading), e) combined two-joint representation (dark shading) and single-joint representation (light shading). The hip joint angle corresponds to the posterior hip joint angle, an angle measured in a posterior direction between the line of the trunk and thigh.

Table 5. Hip extension torque generator parameters

\begin{tabular}{cccc}
\hline parameter & mono & $\begin{array}{c}\text { single } \\
\text { joint }\end{array}$ & $\begin{array}{c}\text { bounds } \\
(\mathrm{LB}-\mathrm{UB})\end{array}$ \\
\hline $\mathrm{T}_{\max }$ & 133.44 & 199.08 & $1.4\left(\mathrm{~T}_{0}\right)$ \\
$\mathrm{T}_{0}$ & 95.31 & 142.20 & $0.8-1.2\left(\mathrm{~T}_{0}\right)$ \\
$\omega_{\max }$ & 12.90 & 11.87 & $11.6-15.4$ \\
$\omega_{\mathrm{c}}$ & 3.44 & 5.81 & $0.15-0.5\left(\omega_{\max }\right)$ \\
$\mathrm{k}_{2}$ & 0.50 & 0.37 & $0.2-2.0$ \\
$\theta_{\mathrm{opt}}$ & 4.56 & 4.85 & $2.7-5.4$ \\
$\mathrm{a}_{\min }$ & 0.99 & 0.94 & $0.2-1.0$ \\
$\mathrm{~m}$ & 0.02 & 0.31 & $0.0-1.0$ \\
$\omega_{1}$ & -1.27 & -0.61 & $-0.5-3.0$
\end{tabular}

Nomenclature and bounds based upon: maximum eccentric torque $\mathrm{T}_{\max }(\mathrm{Nm})$ (Dudley et al.,1990; Webber and Kriellers, 1997), maximum isometric torque $\mathrm{T}_{0}(\mathrm{Nm})$, maximum concentric velocity $\omega_{\max }\left(\mathrm{rad} . \mathrm{s}^{-1}\right)$ (King et al. (2006)), vertical asymptote $\left(\omega=-\omega_{c}\right) \omega_{c}$ $\left(\operatorname{rad} . \mathrm{s}^{-1}\right)$ (UB - Scovil and Ronsky (2006); LB - Umberger et al. (2006)), width of torque-angle relationship $\mathrm{k}_{2}$, optimum angle, $\theta_{\mathrm{opt}}$ (rad) (UB permitted outside joint range where curve may be ascending only), minimum muscle activation, amin, activation rate, $\mathrm{m}$ and point of inflexion $\omega_{1}\left(\mathrm{rad} . \mathrm{s}^{-1}\right)$ (Amiridis et al. (1996)). 


\section{DISCUSSION}

This study has established the contribution of monoarticular hip flexors and hip extensors to total hip flexor and total hip extensor joint torques for an individual and has investigated how calculated torques differ between a single-joint and two-joint approach to modelling hip joint torques. The single-joint and two-joint representations were able to appropriately represent each torque data set with RMSD's of $6-8 \%$ of maximum torque. This level of agreement is similar to other studies in the literature (King and Yeadon, 2002; Forrester et al., 2011) and suggests that either approach may be appropriate.

The maximum voluntary torque contributions from monoarticular muscles to hip flexion (Figure 4a) and hip extension (Figure 5a) vary as a function of hip angle and angular velocity. Considering the two-joint representations, the majority of the contribution to hip flexion torque was from monoarticular muscles (Figure 4), while for hip extension the contributions from biarticular and monoarticular muscles were more even (Figure 5). Literature values (Appendix A) suggest that the contribution from biarticular muscles to hip flexion is larger than found in the current study, although Herzog and Ter Keurs (1988) found an $11-14 \%$ contribution of biarticular muscles to total hip flexion torque, in line with the results found in this study, and probably reflects differences in operating lengths. The strength of the current subject-specific modelling approach is that it benefits from the use of the participants own torque measurements to identify the characteristics of the torque-angle relationship and therefore represent inter-individual differences in the operating lengths. To account for the possibility of different operating lengths, sufficiently wide but realistic bounds are required for these parameters, selecting them based on the upper and lower values found in the literature with some scope for individual variation, prior to determining them by optimisation..

When the knee joint angle was perturbed (extended) from the $90^{\circ}$ used in the data collection, the single-joint representation calculated different hip joint torques to the two-joint approach. For hip flexion the differences were relatively small (around $9 \%$ of maximum torque) for an extended knee whilst for hip extension the differences were substantial (around 33\% of maximum torque). The premise that two joint kinematics affect the torque at a joint is based on the well-established anatomical features of the human musculoskeletal system. Thus caution should be taken when using single-joint representations to calculate torques for a set of two joint kinematics that differ from those used to generate their parameters. For situations where the secondary joint angles are different or changing throughout the movement a two-joint representation is likely to provide improved accuracy, although this requires further examination using a simulation modelling approach. The results of this study are consistent with previous studies at the ankle and knee where a two-joint representation was needed if there were secondary joint movements (Lewis et al., 2012; King et al., 2012). The difference in the capacity of the single and two-joint representations to produce torque within the range of realistic joint kinematics was investigated by considering the kinematics for three jumping movements. The difference between the maximal torques which might occur demonstrated that a single-joint hip flexor would be able to produce around $10 \%$ additional torque, while a single-joint hip extensor about $70 \%$ of the maximum torque exerting capacity of the two-joint representation. In vivo the contractile element force, which generates the net joint moment, is affected by the passive structures in parallel and in series with 
the contractile element and as such the length and velocity of the contractile component would vary depending on the properties of the tissues and the activation state of the muscle fibres. Therefore, the specific contractile element kinematics and subsequent joint torques during jumping may differ slightly from those reported here. The jumping kinematics represent just one example of a multi-joint functional movement, however the multi-joint kinematics effect on two-joint torque representations would also exist to some extent in activities such as running. Whilst the magnitude of joint velocity may differ between jumping and running, there exist periods of concurrent hip flexion and knee extension, and concurrent knee flexion with hip extension (an example of the multi-joint kinematics of running can be found in Riley et al., 2008). Under these conditions, the shortening velocities of the biarticular hip extensors and biarticular hip flexors respectively would be reduced. The findings of this study and those of King et al. (2012) indicate that this could increase torque at the hip and knee joints.

Torque-driven models with single-joint torque representations have typically been used for activities with relatively short contact times $(<200 \mathrm{~ms})$ and relatively small changes in joint kinematics (e.g. tumbling takeoff, King and Yeadon, 2004; high jumping takeoff, Wilson et al., 2007; triple jumping takeoffs, Allen et al., 2010). For these models, although two-joint representations may be able to make slight improvements to the accuracy of single-joint representations, the simulation models have worked appropriately with subject-specific single-joint torque generators. In the future, simulating multi-joint activities with large changes in joint kinematics is likely to benefit from using torque generators consisting of two-joint representations so that the effect of biarticular muscles is appropriately represented. The current alternative would be to use muscle-driven simulation models although these currently require a greater number of parameters of which some could not be accurately determined in a subject-specific manner and therefore the simulation model could not be evaluated appropriately (King and Yeadon, 2015).

The task of establishing the contribution of different muscles to the total torque produced at a joint is non-trivial and ultimately with current technology requires some assumptions to be made. In this study surface EMG measurements were taken and used with previously determined biarticular strength parameters to estimate the active biarticular torque contribution during maximal voluntary hip flexion and hip extension trials with a fixed knee angle. The biarticular contribution varied from as much as $95 \mathrm{Nm}$ to zero depending on the hip and knee angles and velocities (Figure 1). In the future, it may be possible to isolate and electrically stimulate individual muscles in-vivo to establish specific contributions to joint torques. This sort of technique might offer the potential to improve the quality of the data collected on an individual and lead to more accurate torque representations. Some readers may consider the single participant design used in this study as a limitation. However, while the magnitude of the strength parameters may vary between individuals, the relationships obtained are based on the mechanical properties of the general human musculoskeletal system. Moment arms have been shown to change as a function of joint angle (Grieve et al., 1978; Spoor et al., 1990; Visser at al., 1990; Klein et al., 1996). The single ratio used to calculate the magnitude of biarticular torques acting at the hip joint was derived in a previous study (King et al., 2012) and does not represent a variable moment arm but rather the mean relationship of moment arms at two joints for a variety of joint configurations. The study of Out et al. (1996) demonstrated that a simulation of muscle moments is more sensitive to the mean 
moment arm value than to the specific nature of how the moment arm changes with joint angle. In the present study, whilst the relationship between moment arm and joint angle is not defined its strength lies is in the identification of a subject-specific mean value for the moment arms of two joints using strength measurements. It is possible to derive subject-specific parameters for a muscle-driven simulation from excursion-based methods and/or imaging techniques although it is more common to use parameters gathered from the literature. When selecting moment arms from the literature they may not represent one or other of the moment arms of a biarticular muscle or the mean relationship between both joints. In the case of the representations in this study they are guided by a solution space informed by the literature, but the actual values are derived from the least squares fit to the participant's own experimental torque data and not chosen arbitrarily. This participant was found to have strength characteristics of the hip flexors and hip extensors that agree with estimates found elsewhere in the literature. Adding additional subjects to this study would add additional information on how strength varies between individuals (not an aim of this study) but would not change the conclusions of this study.

In conclusion, isovelocity torque - angle - angular velocity data can be used to establish the contribution from monoarticular hip flexor and hip extensor muscles with both single-joint and two-joint representations both able to fit the data. To account for biarticular effects when a secondary joint angle changes implies a two-joint torque representation. This approach overcomes a limitation of single-joint representations, which may be overly simple while building on the strength of determining subjectspecific parameters from measurements on the participant rather than relying on data from the literature. Consequently, whole body movements that experience large changes to the joint kinematics of adjacent joints where biarticular muscles operate, such as concurrent hip and knee extension of approximately $90^{\circ}$, may necessitate increased complexity to model joint torques. Existing torque driven simulation models therefore with short duration and small variation in joint kinematics are likely to be minimally affected by two-joint kinematics even if the biofidelity of the representation of torques is more anatomically accurate.

\section{FUNDING}

This research did not receive any specific grant from funding agencies in the public, commercial, or not-for-profit sectors. 


\section{REFERENCES}

Allard, P., Stokes, I.A.F., \& Blanchi, J. (1995). Three-dimensional analysis of human movement. Human Kinetics Publishers, Champaign.

Allen, S.J., King, M.A., \& Yeadon, M.R. (2010). Is a single or double arm technique more advantageous in triple jumping? Journal of Biomechanics, 43, 3156-3161.

Allen, S.J., King, M.A., \& Yeadon, M.R. (2013). Trade-offs between horizontal and vertical velocities during triple jumping and the effect on phase distances. Journal of Biomechanics, 46, 979-983.

Amiridis, I.G., Martin, A., Morlon, B., Martin, L., Cometti, G., Pousson, M., \& van Hoecke, J. (1996). Co-activation and tension-regulating phenomena during isokinetic knee extension in sedentary and highly skilled humans. European Journal of Applied Physiology, 73, 149-156.

Anderson, F.C., \& Pandy, M.G. (1999). A dynamic optimisation solution for vertical jumping. Computer Methods in Biomechanics and Biomedical Engineering, 2, 201231.

Arnold, E. M., Ward, S. R., Lieber, R. L., \& Delp, S. L. (2010). A model of the lower limb for analysis of human movement. Annals of biomedical engineering, 38, 269279.

Bohm, H., Cole, G.K., Bruggemann, G.P., \& Ruder, H. (2006). Contribution of muscle series elasticity to maximum performance in drop jumping. Journal of Applied Biomechanics, 22, 3-13.

Corana, A., Marchesi, M., Martini, C., \& Ridella, S. (1987). Minimizing multimodal functions of continuous variables with the "simulated annealing" algorithm. ACM Transactions on Mathematical Software, 13, 262-280.

Davis, R.B., Ounpuu, S., Tyburski, D., \& Gage, J.R. (1991). A gait analysis data collection and reduction technique. Human Movement Science, 10, 575-587.

Domire, Z. J., \& Challis, J. H. (2007). The influence of squat depth on maximal vertical jump performance. Journal of sports sciences, 25, 193-200.

Duda, G.N., Brand, D., Freitag, S., Lierse, W., \& Schneider, E. (1996). Variability of femoral muscle attachments. Journal of Biomechanics, 29, 1185-1190.

Dudley, G.A., Harris, R.T., Duvoisin, M.R., Hather, B.M., \& Buchanan, P. (1990). Effect of voluntary vs. artificial activation on the relationship of muscle torque to speed. Journal of Applied Physiology, 6, 2215-2221.

Forrester, S.E., Yeadon, M.R., King, M.A., \& Pain, M.T.G. (2011). Comparing different approaches for determining joint torque parameters from isovelocity dynamometer measurements. Journal of Biomechanics, 44, 955-961.

Grieve, D. W., Pheasant, S., and Cavanagh, P. R., Prediction of Gastrocnemius Length from Knee and Ankle Joint Posture, University Park Press, Baltimore, pp. 405-412, 1978.

Herzog, W., \& Ter Keurs, E.D.J. (1988). Force-length relation of in-vivo human rectus femoris muscles. Pflugers Archive European Journal of Physiology, 411, 642-647.

Jacobs, R., Bobbert, M. F., \& van Ingen Schenau, G. J. (1996). Mechanical output from individual muscles during explosive leg extensions: the role of biarticular muscles. Journal of Biomechanics, 29, 513-523.

Katsavelis, D., \& Threlkeld, A.J. (2014). Quantifying thigh muscle co-activation during isometric knee extension contractions: Within- and between-session reliability. Journal of Electromyography and Kinesiology : Official Journal of the International Society of Electrophysiological Kinesiology, 24, 502-507.

King, M.A., \& Yeadon, M.R. (2002). Determining subject-specific torque parameters for use in a torque-driven simulation model of dynamic jumping. Journal of Applied Biomechanics, 18, 207-217. 
King, M.A., \& Yeadon, M.R. (2004). Maximising somersault rotation in tumbling. Journal of Biomechanics, 37, 471-477.

King, M.A., \& Yeadon, M.R. (2015). Advances in the development of whole body computer simulation modelling of sports technique. Movement \& Sport Sciences Science \& Motricité, 90, 55-67.

King, M.A., Kong, P.W., \& Yeadon, M.R. (2005). Evaluation of a torque driven computer simulation model of springboard diving. Proceedings of the $X$ th International Symposium on Computer Simulation in Biomechanics, Case Western Reserve University, 59-60.

King, M.A., Wilson, C., \& Yeadon, M.R. (2006). Evaluation of a torque-driven model of jumping for height. Journal of Applied Biomechanics, 22, 264-274.

King, M.A., Lewis, M.G.C., \& Yeadon, M.R. (2012). Is it necessary to include biarticular effects within joint torque representations of knee flexion and knee extension? International Journal for Multiscale Computational Engineering, 10, 117-130.

Klein, P., Mattys, S., and Rooze, M., Moment arm length variations of selected muscles acting on talocrural and subtalar joints during movement: An in vitro study, J. Biomech., vol. 29, no. 1, pp. 21-30, 1996.

Kooistra, R.D., de Ruiter, C.J., \& de Haan, A. (2007). Conventionally assessed voluntary activation does not represent relative voluntary torque production. European Journal of Applied Physiology, 100, 309-320.

Lewis, M.G.C., King, M.A., Yeadon, M.R. \& Conceição, F. (2012). Are joint torque models limited by an assumption of monoarticularity? Journal of Applied Biomechanics, 28, 520-529.

Neptune, R.R., \& Hull, M.L. (1998). Evaluation of performance criteria for simulation of submaximal steady-state cycling using a forward-dynamic model. Journal of Biomechanical Engineering, 120, 334-341.

Out, L., Vrijkotte, T. G. M., van Soest, A. J., and Bobbert, M. F., Influence of the parameters of a human triceps surae muscle model on the isometric torque-angle relationship, J. Biomech. Eng., vol. 118, pp. 17-25, 1996.

Pandy, M.G., Zajac, F.E., Sim, E., \& Levine, W.S. (1990). An optimal control model for maximum height human jumping. Journal of Biomechanics, 23, 1185-1198.

Pavol, M.J., \& Grabiner, M.D. (2000). Knee strength variability between individuals across ranges of motion and hip angles. Medicine and Science in Sports and Exercise, 32, 985-992.

Riley, P.O., Dicharry, J., Franz, J., Croce, U.D., Wilder, R.P. \& Kerrigan D.C. (2008). A kinematics and kinetic comparison of overground and treadmill running. Medicine and Science in Sports and Exercise, 40, 1093-1100.

Schwartz, F.P., Bottaro, M., Celes, R.S., Brown, L.E., \& Nascimento, F.A.O. (2010). The influence of velocity overshoot movement artifact on isokinetic knee extension tests. Journal of Sports Science and Medicine, 9, 10-146

Scovil, C.Y., \& Ronsky, J.L. (2006). Sensitivity of a Hill-based muscle model to perturbations in model parameters. Journal of Biomechanics, 39, 2055-2063.

Soest, A.J. van, Schwab, A.L., Bobbert, M.F., \& Ingen Schenau, G.J. van (1993). The influence of the biarticularity of the gastrocnemius muscle on vertical jumping achievement. Journal of Biomechanics, 26, 1-8.

Spoor, C. W., van Leewun, J. L., Meskers, C. G., Titulaer, A. F., and Huson, A., Estimation of instantaneous moment arms of the lower-leg muscles, J. Biomech., vol. 23, no. 12, pp. 1247-1259, 1990.

Umberger, B.R., Gerritsen, K.G.M., \& Martin, P.E. (2006). Muscle fibre type effects on energetically optimal cadences in cycling. Journal of Biomechanics, 39, 14721479. 
Visser, J. J., Hoogkamer, J. E., Bobbert, M. F., and Huijing, P. A., Length and moment arm of human leg muscles as a function of knee and hip-joint angles, Eur. J. Appl. Physiol. Occupat. Physiol., vol. 61, no. 5-6, pp. 453-460, 1990.

Webber, S., \& Kriellers, D. (1997). Neuromuscular factors contributing to in vivo eccentric moment generation. Journal of Applied Physiology, 83, 40-45.

Wilson, C., Yeadon. M.R., \& King, M.A. (2007). Considerations that affect optimised simulation in a running jump for height. Journal of Biomechanics, 40, 3155-3161.

Winter, D.A. (1990). Biomechanics and motor control of human movement. Wiley, New York, pp. 277.

Wood, G.A., \& Jennings, L.S. (1979). On the use of spline functions for data smoothing. Journal of Biomechanics, 12, 447-479.

Yeadon, M.R. (1990). The simulation of aerial movement - I. The determination of orientation angles from film data. Journal of Biomechanics, 23, 59-66. 


\section{APPENDIX A}

For any muscle (i), by assuming a uniform strength per unit area of physiological cross sectional area (PCSA) and identifying from the literature that muscle's fibre pennation angle (ai) and moment arm (di), then a muscles unit-less maximum isometric torque contribution (Ti) at optimal muscle length can be calculated from:

$$
T_{i}=d_{i} \cdot P C S A_{i} \cdot \cos \alpha_{i}
$$

And therefore its percentage contribution to the net torque generated by $n$ muscles is:

$$
\% \text { Contribution }_{i}=\frac{T_{i}}{\sum_{i=1}^{n} T_{i}} \cdot 100
$$

The muscles shown in Tables $\mathrm{A} 1$ and Table $\mathrm{A} 2$ are grouped according to the joints they cross, such that they are classified as biarticular or monoarticular. If a muscle crosses two joints, but only one of these is included in the two-joint representation of joint torque, then it is classified as a monoarticular muscle for the purposes of this paper.

\begin{tabular}{|c|c|c|c|c|}
\hline muscle & 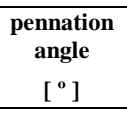 & $\begin{array}{c}\text { moment } \\
\text { arm at hip } \\
{[\mathrm{mm}]} \\
\end{array}$ & $\begin{array}{l}\text { PCSA } \\
{\left[\mathbf{m m}^{2}\right]}\end{array}$ & $\begin{array}{c}\text { contribution } \\
{[\%]}\end{array}$ \\
\hline rectus femoris & 10 & 22.0 & 3357 & 37.6 \\
\hline \multicolumn{4}{|c|}{$\begin{array}{l}\% \text { contribution from biarticular muscles acting about the hip } \\
\text { and knee }\end{array}$} & 37.6 \\
\hline iliacus & 5 & 14.8 & 1817 & 13.8 \\
\hline adductor longus & 4 & 14.8 & 1518 & 11.6 \\
\hline sartorius & 0 & 60.0 & 365 & 11.3 \\
\hline tensor fasciae latae & 2 & 41.8 & 516 & 11.1 \\
\hline psoas major & 5 & 14.8 & 1383 & 10.5 \\
\hline pectineus & 0 & 14.8 & 535 & 4.1 \\
\hline \multicolumn{4}{|c|}{$\begin{array}{l}\% \text { contribution by monoarticular muscles (any hip flexor not } \\
\text { acting about the knee joint) }\end{array}$} & 62.4 \\
\hline
\end{tabular}

Table A1. Contributions to hip flexion torque

PCSA and pennation angles from Allard et al. (1995), moment arm values from Duda et al. (1996) and Jacobs et al. (1996). The moment arm of the rectus femoris was selected for a cadaver specimen that most resembled the same moment arm ratio calculated for the participant in this study. Sartorius was grouped as a monoarticular hip flexor because of its small moment arm at the knee. 
Table A2. Contributions to hip extension torque

\begin{tabular}{|c|c|c|c|c|}
\hline 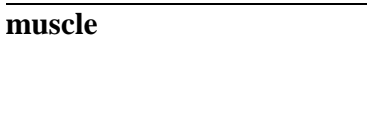 & $\begin{array}{r}\text { pennation } \\
{\left[^{\circ}\right]}\end{array}$ & $\begin{array}{l}\underset{\text { hip }}{\text { ment arm at }} \\
{[\mathrm{mm}]}\end{array}$ & $\begin{array}{c}\text { PCSA } \\
{\left[\mathrm{mm}^{2}\right]}\end{array}$ & $\begin{array}{c}\text { contribution } \\
{[\%]}\end{array}$ \\
\hline biceps femoris (long head) & 7 & 46.7 & 2881 & 21.8 \\
\hline semitendinosus & 4 & 52.7 & 938 & 8.1 \\
\hline semimembranosus & 15 & 39.3 & 3988 & 24.7 \\
\hline \multicolumn{4}{|c|}{$\%$ contribution from biarticular muscles acting about the hip and knee } & 54.6 \\
\hline gluteus maximus superficial & 0 & 50.8 & 2185 & 18.1 \\
\hline adductor magnus middle & 3 & 63.8 & 1362 & 14.2 \\
\hline adductor magnus posterior & 3 & 47.8 & 1674 & 13.1 \\
\hline
\end{tabular}

PCSA and pennation angles from Allard et al. (1995), moment arm values from Duda et al. (1996) and Jacobs et al. (1996). 


\section{APPENDIX B}

Single-joint nine parameter torque representation

The torque-velocity relationship was taken from Yeadon et al. (2006) and the torqueangle relationship from King et al. (2006). The maximal voluntary torque T for any joint velocity $\omega$ and joint angle $\theta$ was calculated using 9 parameters where these represented the tetanic torque $\mathrm{T}(4)$, multiplied by differential activation a(3), multiplied by a fraction of the maximum torque available at any velocity for a given component angle $\mathrm{T} \theta(2)$.

$$
\mathrm{T}=\mathrm{T}_{(4) .} \mathrm{a}_{(3)} \cdot \mathrm{T}_{\theta(2)} \text { where: }
$$

\section{Two-joint representation of knee joint torques}

The 19-parameter two-joint torque generator function for calculating knee extension and knee flexion torques in King et al. (2012) included both monoarticular and biarticular representations and expressed maximum voluntary torque as a function of knee joint angle $\theta k$, hip joint angle $\theta \mathrm{h}$ and the corresponding two angular velocities $\omega \mathrm{k}$ and wh. The 19-parameter function consisted of the sum of a nine-parameter monoarticular function of $\theta \mathrm{k}$ and $\omega \mathrm{k}$ which was identical to the single-joint nineparameter representation, and a ten-parameter biarticular function of $\theta \mathrm{k}, \theta \mathrm{h}$, wh and wh. The ten-parameter biarticular function was based on the nine-parameter function and was the function of a calculated biarticular component angle $\theta \mathrm{Bi}$ and biarticular component angular velocity $\omega \mathrm{Bi}$, with one additional parameter $\mathrm{R}$ describing the ratio of moment arms at the knee $\mathrm{dk}$ and hip dh joints:

$$
\mathrm{R}=\frac{\mathrm{d}_{\mathrm{h}}}{\mathrm{d}_{\mathrm{k}}}
$$

This extra parameter allowed $\theta \mathrm{K}$ to be added to $\theta \mathrm{H}$ in a meaningful way so that the combined angle represented the 'length' of the biarticular component, where:

$$
\theta_{\mathrm{Bi}}=\theta_{\mathrm{k}}+\mathrm{R} \theta_{\mathrm{h}}
$$

In the same way the ratio $R$ was used to allow wh to be added to wk to give a biarticular component angular velocity, where:

$$
\omega_{\mathrm{Bi}}=\omega_{\mathrm{k}}+\mathrm{R} \omega_{\mathrm{h}}
$$

This resulted in a nine-parameter biarticular function of $\theta \mathrm{Bi}$ and $\omega \mathrm{Bi}$ which took the same form as that of the single-joint representation and monoarticular representation with a torque-velocity and torque-angle representation. The total knee joint torque was calculated as the sum of monoarticular and biarticular torques.

\section{Calculation of biarticular hip torque}

The internal muscle force $\mathrm{Fb}$, associated with the biarticular muscles creating a moment at the knee joint can be calculated from the muscles moment arm at that joint $\mathrm{dk}$, and the torque generated at that joint Tk.

$$
\mathrm{F}_{\mathrm{b}}=\frac{\mathrm{T}_{\mathrm{k}}}{\mathrm{d}_{\mathrm{k}}}
$$


The internal muscle force will generate a moment at the hip joint Th, associated with this internal muscle force and the moment arm of the biarticular muscles at the hip joint dh.

$$
\mathrm{T}_{\mathrm{h}}=\mathrm{F}_{\mathrm{b}} \cdot \mathrm{d}_{\mathrm{h}}
$$

Combining the equations for $\mathrm{Fb}$ and Th shows how in a whole-body torque-driven simulation model incorporating two-joint torque generators the hip joint torque generated by the biarticular knee-hip components can be calculated from the torque they generate at the knee joint and their moment arm ratio:

$$
\mathrm{T}_{\mathrm{h}}=\mathrm{T}_{\mathrm{k}} \cdot \frac{\mathrm{d}_{\mathrm{h}}}{\mathrm{d}_{\mathrm{k}}} \quad \text { therefore } \mathrm{T}_{\mathrm{h}}=\mathrm{T}_{\mathrm{k}} \cdot \mathrm{R}
$$




\section{Appendix C - Measurement of two-footed jump, lower limb kinematics:}

Hip and knee joint kinematics were measured for three different types of two-footed jump: a) Maximal height squat jump - starting from a stationary position with knees bent to approximately $90^{\circ}$ posterior knee joint angle, b) Maximal height countermovement jump - participant started from a standing posture with their hands by their side, c) Maximal broad jump (two-footed standing long jump). For all jumps the participant was allowed to use their arm to assist with the jump but encouraged to keep all arm movements in the transverse plane.

Each type of jump was completed 4 times, with the best performance selected for further analysis; maximal height for squat and countermovement jumps and maximum horizontal distance for the broad jump. A 16 camera (M2 MCam) Vicon Motion Analysis System (OMG PIc, Oxford, UK) sampling at $300 \mathrm{~Hz}$ was used to track the location of markers placed on left and right limbs sides of the body over known bony landmarks (King et al., 2012). The trunk segment was defined by a line extending between the midpoint of markers placed laterally of the left and right shoulder joint centre, to the midpoint of hip joint centres, calculated from the locations of the anterior superior iliac spine and posterior superior iliac spine markers (Davis et al., 1991). The thigh was represented by a line between the hip joint centre and the midpoint between pairs of markers placed on the medial and lateral sides of the knee joint. The shank segment was defined by a line passing from the knee joint centre to the centre of pairs of markers placed on the lateral and medial malleoli of the associated limb. The knee and hip joint angles were calculated from the joint centre co-ordinates (Yeadon, 1990) and quintic splines (Wood and Jennings, 1979) were fitted to the joint angle time histories. Hip and knee joint angles were calculated as the average of left and right limbs for further analysis of jumping. 